\title{
Disparities in the Use of Continent Urinary Diversions after Radical Cystectomy for Bladder Cancer
}

\author{
Nicholas J. Farber ${ }^{\mathrm{a}, *}$, Izak Faiena ${ }^{\mathrm{a}}$, Viktor Dombrovskiy ${ }^{\mathrm{b}}$, Alexandra L. Tabakin ${ }^{\mathrm{a}}$, Brian Shinder ${ }^{\mathrm{a}}$, \\ Rutveej Patel ${ }^{\mathrm{a}}$, Sammy E. Elsamra ${ }^{\mathrm{c}}$, Thomas L. Jang ${ }^{\mathrm{c}}$, Eric A. Singer ${ }^{\mathrm{c}}$ and Robert E. Weiss ${ }^{\mathrm{c}}$ \\ ${ }^{a}$ Division of Urology, Rutgers Robert Wood Johnson Medical School, New Brunswick, NJ, USA \\ ${ }^{\mathrm{b}}$ Department of Surgery, Rutgers Robert Wood Johnson Medical School, New Brunswick, NJ, USA \\ ${ }^{\mathrm{c}}$ Section of Urologic Oncology, Rutgers Cancer Institute of New Jersey, New Brunswick, NJ, USA
}

\begin{abstract}
.
Background: Radical cystectomy (RC) with ileal conduit (IC) or continent diversion (CD) is standard treatment for high-risk non-invasive and muscle-invasive bladder cancer.

Objective: Our aim is to study contemporary trends in the utilization of ICs and CDs in patients undergoing RC.

Methods: Using the National Inpatient Sample 2001-2012, we identified all patients diagnosed with a malignant bladder neoplasm who underwent RC followed by IC or CD. Patient demographics, comorbidities, length of stay (LOS), and inhospital complications, mortality, and costs were compared. Multivariable logistic regression analysis, Chi square, and $t$-tests were used for analysis.

Results: Between 2001-2012, approximately 69,049 ICs and 6,991 CDs were performed. CDs increased from 2001 to 2008, but declined after $2008(p<0.0001)$. Patients of all ages received ICs at a higher rate than CDs $(40-59$ years: $79.5 \%$ vs. $20.5 \%$; $60-69$ years: $88.0 \%$ vs. $12.0 \% ; p<0.0001)$. There was a difference in males vs. females $(10.2 \%$ vs. $4.0 \%$; OR 2.36$)$ and Caucasians vs. African Americans (9.0\% vs. 6.7\%; OR 1.49) when comparing CD rates. CD rates were highest in the West, urban teaching centers, and large hospitals $(p<0.001)$. ICs were associated with higher rates of overall postoperative complications $(p=0.0185)$ including infection $(p=0.002)$ and mortality $(p<0.0001)$. In-hospital costs were greater for the CD group.

Conclusions: The number of CDs has declined recently. Patients of all ages are more likely to receive ICs than CDs. Gender, racial, and geographic disparities exist among those receiving CDs. CDs are associated with lower rates of in-hospital complications and mortality, but higher in-hospital costs.
\end{abstract}

Keywords: Radical cystectomy, urinary diversion, ileal conduit, neobladder, trends, disparity

\section{INTRODUCTION}

Every year, approximately 16,870 people die of bladder cancer, making it the 9 th most deadly cancer

\footnotetext{
${ }^{*}$ Correspondence to: Nicholas J. Farber, MD, Division of Urology, 1 RWJ Place, MEB Suite 584A, New Brunswick, NJ 08901, USA. Tel.: +1 732235 8853; Fax: +1 732235 8018; E-mail: nfarber@gmail.com.
}

in the United States [1]. For high-risk non-invasive and muscle-invasive bladder cancer, radical cystectomy (RC) is a standard treatment, followed by either an ileal conduit (IC) or a continent diversion (CD). Various patient-related factors such as age, functional status, preoperative tumor stage, preoperative chemotherapy, socioeconomic status, renal function, and other medical comorbidities are taken 
into account when selecting a urinary diversion type [2]. Further, it is necessary to consider perioperative factors including technical feasibility of the surgery, postoperative complications rates, cost, postoperative health-related quality of life, and patient ability to care for their diversion. Currently, no randomized studies compare IC and CD, and there is no consensus as to which type of urinary diversion provides better functional or quality of life outcomes [2, 3].

Although many bladder cancer experts support CD as the standard of care in select patients and advocate the use of continent diversions in older patients $[2,4]$, the trend in continent diversion rate seems to be declining at many centers $[5,6]$. Prior studies have suggested that inadequate training among urologists in performing neobladder construction [7], the increased time required for neobladder creation, and higher complication rates in neobladder construction [2] may contribute to the decline in CD. Others have reported that younger, privately insured men who undergo surgery at an urban teaching hospital are more likely to receive a $\mathrm{CD}$, implying that gender, socioeconomic, and geographic disparities may influence diversion type [2]. Further elucidating trends in urinary diversion can guide us in identifying inequalities and barriers to bladder cancer care. Therefore, our aim is to study contemporary trends in the utilization of ICs and CDs in patients undergoing radical cystectomy.

\section{MATERIALS AND METHODS}

\section{Data source}

Following approval from the Rutgers Robert Wood Johnson Medical School Institutional Review Board, we accessed the Nationwide/National Inpatient Sample (NIS), the largest all-payer inpatient database that includes information on approximately $20 \%$ of inpatient stays in the acute care community hospitals in the United States. The most current issue of the NIS contains approximately 250 data elements such as patient demographics, hospital characteristics, primary and secondary diagnoses and procedures, AHRQ (Agency for Healthcare Research and Quality) comorbidity measures, total hospital charge and length of stay, and other healthcare related variables. Using supplemental Cost-to Charge Ratio Files, total hospital charge may be converted to total hospital cost that improves analysis of hospital resource utilization. Database sampling strategy allows calculation of national estimates.

\section{Study cohort and covariates}

Using the International Classification of Diseases, Ninth Revision, Clinical Modification (ICD-9-CM), we identified in the NIS 2001-2012 all patients aged 40 years or older with the principal diagnosis of malignant neoplasm of bladder (diagnosis codes 188.x or 233.7) who underwent radical cystectomy (procedure code 57.71) followed by either ileal conduit (56.61) or orthotopic neobladder/continent diversion (57.87).

Once categorized by the urinary diversion type, we analyzed and compared postoperative outcomes in these groups taking into account patient and hospital characteristics. Patient demographic characteristics included age, gender, race (white, black, Hispanic, other/missing), and comorbidities. Hospital characteristics included hospital geographic region (Northeast, Midwest, South, West), hospital teaching status (rural, urban non-teaching, urban teaching), and hospital size (small, medium, large). Using the appropriate ICD-9-CM diagnosis codes we identified and analyzed the following in-hospital postoperative complications: cardiac complications including myocardial infarction (997.1, 410.00-410.02, 410.10$410.12,410.20-410.22,410.30-410.32,410.40-41$ 0.42, 410.50-410.52, 410.60-410.62, 410.70-410. $72,410.80-410.82,410.90-410.92$, and 427.5), respiratory complications including pneumonia $(997.3 \mathrm{x}$, 507.0, 512.1, 512.8, 518.4, 518.5, 518.81-518.82, 480.x, 481, 482.x, 483.x, 485, 486), renal complications $(997.5,584 . x, 593.81)$, urinary tract infection (599.0 and 996.64), sepsis and bloodstream infection (038.xx, 995.91, 995.92, 785.52, 996.61-996.62, 998.0, 998.59, and 999.3x), surgical site infection and non-healing surgical wound $(998.5 \mathrm{x}$, 998.30-998.32, 998.83), C. difficile (008.45), and postoperative bleeding (998.11-998.12, 285.1). Other analyzed in-hospital postoperative outcomes included hospital mortality, length of hospital stay (LOS), and total cost of stay.

\section{Statistical analysis}

SAS software, version 9.4 (SAS Institute, Cary, NC) was used for data analysis and all statistics. Intergroup differences for categorical variables were tested by Chi square test following by the multivariable logistic regression analysis with control for possible confounders: patient demographic characteristics (age, gender and race), comorbidities, and hospital characteristics (location, size and teaching 


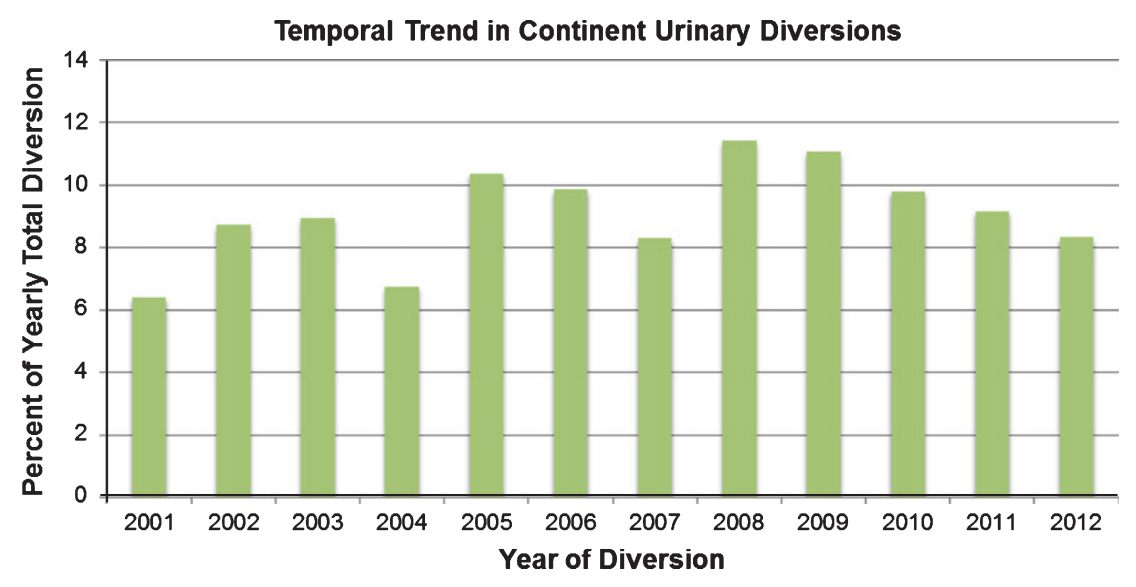

Fig. 1. Trends in percentage of continent urinary diversions from 2001-2012.

status). Trend in the use of both surgical procedures from 2001 to 2012 was analyzed with the CochranArmitage trend test. Continuous variables were tested for normality of distribution and compared using Student's $t$-test or Wilcoxon rank sum test, as appropriate. To control for confounders above, LOS and cost were also compared using the generalized linear modeling approach (SAS GENMOD procedure with gamma model with log link). Cost data for various years were adjusted for inflation to cost data 2012. $P<0.05$ was considered statistically significant for all tests.

\section{RESULTS}

There were 76,040 cases that met the inclusion criteria. The estimated national total number of IC performed was 69,049 (90.8\%) and the total number of $\mathrm{CD}$, including orthotopic neobladders, was 6,991 (9.2\%) (Fig. 1). The absolute number of CDs gradually increased since 2001 , with a statistically significant trend test $(p<0.0001)$. However, the peak number of CDs was seen in 2008, and then the total number of CDs has decreased every year thereafter.

Demographic characteristics of patients in two surgical groups are presented in Table 1. On univariate analysis, patients with age 40-59 represented the largest proportion of individuals who received a CD $(40.4 \%, p<0.0001)$; this percentage decreased as the age increased. Males were 2.69 times as likely as females to receive CD (OR 2.69, 95\% CI: 2.46-2.96, $p<0.001$ ). In terms of race, both Caucasian (OR 1.39, 95\% CI: $1.20-1.62, p<0.0001)$ and Hispanic (OR 1.59, 95\% CI: $1.29-1.96, p<0.0001)$ patients were more likely to have $\mathrm{CD}$ when compared to African Americans.

Hospital and regional characteristics of the two groups are presented in Table 2. Regionally, CDs were most likely to be performed in the West and are least likely to be performed in the South $(p<0.001)$. With respect to type of hospital, urban teaching hospitals performed the highest proportion of CDs and were more likely than urban non-teaching hospitals to perform CD (OR 1.58, 95\% CI: 1.36-1.84, $p<0.001$ ). Large sized hospitals performed the highest proportion of CDs and were significantly more likely than small sized hospital to perform CD (OR 1.26, 95\% CI: $1.15-1.38, p<0.001$ ).

In the multivariable logistic regression analysis, adjustments for age, gender, race, comorbidities, and hospital characteristics were performed. When adjusting for covariates, demographic predictors of CD were male gender (OR 2.36, 95\% CI 2.15-2.61), Caucasian race compared to African-American race (OR 1.49, 95\% CI 1.273-1.750), and young age (40-59 years) compared to old age ( $>80$ years) (OR 15.782; 95\% CI 13.42-18.57). In terms of hospital characteristics, predictors of $\mathrm{CD}$ were West census region compared to South (OR 2.160; 95\% CI 2.009-2.321), Northeast versus South (OR 1.56; 95\% CI 1.447-1.68), Urban teaching versus non-teaching hospital (OR 2.276; 95\% CI 2.107-2.46), and large versus small hospital bed size (OR 1.31; $95 \%$ CI 1.19-1.45).

Complications and mortality of the two groups are presented in Table 3. On univariate analysis, patients with an IC had a greater rate of postoperative complications overall compared to those with a CD $(52.8 \%$ vs. $44.7 \% ; p<0.0001)$. When stratified by individual complications, IC had higher odds than CD for every 
Table 1

Patient demographic data

\begin{tabular}{|c|c|c|c|}
\hline Characteristic & Ileal Conduit & Continent Diversion & $P$ value \\
\hline Age, years (mean \pm standard deviation) & $69.7 \pm 9.8$ & $61.6 \pm 9.3$ & $<0.0001$ \\
\hline Age groups, years & & & $<0.0001$ \\
\hline $40-59$ & $10,947(79.5 \%)$ & $2,822(20.5 \%)$ & \\
\hline $60-69$ & $19,901(88.0 \%)$ & $2,715(12.0 \%)$ & \\
\hline $70-79$ & $27,287(95.5 \%)$ & $1,293(4.5 \%)$ & \\
\hline $80+$ & $10,912(98.5 \%)$ & $162(1.5 \%)$ & \\
\hline Gender & & & $<0.0001$ \\
\hline Male & $57,098(89.8 \%)$ & $6,487(10.2 \%)$ & \\
\hline Female & $11,941(96.0 \%)$ & $504(4.0 \%)$ & \\
\hline Race & & & $<0.0001$ \\
\hline Caucasian & $48,988(91.0 \%)$ & $4,867(9.0 \%)$ & \\
\hline African-American & $2,604(93.3 \%)$ & $186(6.7 \%)$ & \\
\hline Hispanic & $1,739(89.8 \%)$ & $198(10.2 \%)$ & \\
\hline Other and missing & $15,717(90.0 \%)$ & $1,740(10.0 \%)$ & \\
\hline \multicolumn{4}{|l|}{ Comorbidities } \\
\hline Anemia & $10,216(14.8 \%)$ & $677(9.7 \%)$ & $<0.0001$ \\
\hline Chronic pulmonary disease & $14,374(20.8 \%)$ & $858(12.3 \%)$ & $<0.0001$ \\
\hline Coagulopathy & $2,621(3.8 \%)$ & $192(2.8 \%)$ & $<0.0001$ \\
\hline Congestive heart failure & $3,386(4.9 \%)$ & $127(1.8 \%)$ & $<0.0001$ \\
\hline Diabetes & $13,172(19.1 \%)$ & $975(14.0 \%)$ & $<0.0001$ \\
\hline Hypertension & $36,534(52.9 \%)$ & $3,088(44.2 \%)$ & $<0.0001$ \\
\hline Hypothyroidism & $4,249(6.2 \%)$ & $220(3.2 \%)$ & $<0.0001$ \\
\hline Liver disease & $630(0.9 \%)$ & $101(1.4 \%)$ & $<0.0001$ \\
\hline Metastatic cancer & $11,585(16.8 \%)$ & $978(14.0 \%)$ & $<0.0001$ \\
\hline Neurological disorders & $1,879(2.7 \%)$ & $116(1.7 \%)$ & $<0.0001$ \\
\hline Obesity & $4,193(6.1 \%)$ & $376(5.4 \%)$ & 0.024 \\
\hline Pulmonary vascular disease & $1,180(1.7 \%)$ & $94(0.4 \%)$ & 0.026 \\
\hline Renal failure & $4,676(6.8 \%)$ & $211(3.0 \%)$ & $<0.0001$ \\
\hline Valvular disease & $2,685(3.9 \%)$ & $175(2.5 \%)$ & $<0.0001$ \\
\hline Weight loss & $4,584(6.6 \%)$ & $209(3.0 \%)$ & $<0.0001$ \\
\hline TOTAL & 69,049 & 6,991 & \\
\hline
\end{tabular}

Table 2

Hospital and regional data

\begin{tabular}{lccc}
\hline Characteristic & $\begin{array}{c}\text { Ileal } \\
\text { Conduit }\end{array}$ & $\begin{array}{c}\text { Continent } \\
\text { Diversion }\end{array}$ & $p$ value \\
\hline Census Region & & \multicolumn{2}{c}{$<0.0001$} \\
Northeast & $13,878(89.4 \%)$ & $1,646(10.6 \%)$ & \\
Midwest & $17,733(91.3 \%)$ & $1,701(8.8 \%)$ & \\
South & $23,768(93.1 \%)$ & $1,763(6.9 \%)$ & \\
West & $13,669(87.9 \%)$ & $1,882(12.1 \%)$ & \\
Location & & & $<0.0001$ \\
Rural & $3,073(92.7 \%)$ & $241(7.3 \%)$ & \\
Urban Non-teaching & $17,345(95.3 \%)$ & $859(4.7 \%)$ & \\
Urban teaching & $48,160(89.1 \%)$ & $5,863(10.9 \%)$ & \\
Hospital Bed Size & & & $<0.0001$ \\
Small & $6,330(92.1 \%)$ & $539(7.9 \%)$ & \\
Medium & $12,301(92.1 \%)$ & $1,053(7.9 \%)$ & \\
Large & $49,948(90.3 \%)$ & $5,371(9.7 \%)$ & \\
\hline & & &
\end{tabular}

analyzed complication except surgical site infections. Patients receiving an IC were more likely to die in the hospital than those receiving CD (OR 3.3, 95\% CI 2.46-4.40; $p<0.0001$ ) (Table 4).

In the multivariable logistic regression analysis with adjustment for age, gender, race, comorbidities, and hospital characteristics, patients with IC were 1.07 times as likely as those with $\mathrm{CD}$ to have a complication of any kind (OR 1.07, 95\% CI $1.01-1.12 ; p=0.0185)$ and 1.13 times as likely to have an infectious complication (95\% CI 1.05-1.22; $p=0.002$ ). Similarly, in the logistic regression analysis patients with IC were 1.87 times as likely as those with $\mathrm{CD}$ to die during index hospitalization (OR 1.87, 95\% CI 1.38-2.52; $p<0.0001$ ) (Table 4).

Estimated hospital resource usage can be found in Table 3. On univariate analysis, median cost was significantly greater in the $\mathrm{CD}$ group compared to ICs $(p<0.0001)$, while the IC group had significantly greater median LOS compared to CDs $(p<0.0001)$. When adjusted for age, gender, comorbidities, regional and hospital characteristics, LOS was similar in the two groups (OR 1.02, 95\% CI 0.99-1.05) while cost in the IC group remained less than in CD group (OR 0.95, 95\%CI 0.92-0.98) (Table 4).

\section{DISCUSSION}

Despite various attempts to elucidate the optimal type of urinary diversion following radical 
Table 3

In-hospital complications, mortality, length of stay, and cost

\begin{tabular}{lccr}
\hline Variable & Ileal Conduit & $\begin{array}{c}\text { Continent } \\
\text { Diversion }\end{array}$ & $p$ value \\
\hline Complications, Overall (\%) & 52.8 & 44.7 & $<0.0001$ \\
Infectious & 16.5 & 12.1 & $<0.0001$ \\
Cardiac & 4.8 & 3.6 & $<0.0001$ \\
Respiratory & 11.4 & 7.3 & $<0.0001$ \\
Pneumonia & 3.5 & 2.0 & $<0.0001$ \\
Renal & 11.1 & 9.2 & $<0.0001$ \\
UTI & 5.4 & 3.6 & $<0.0001$ \\
Sepsis & 7.1 & 6.4 & 0.0256 \\
Surgical Site Infection & 3.7 & 3.4 & 0.2618 \\
C. difficile & 2.0 & 1.3 & 0.0001 \\
Bleeding & 19.1 & 15.8 & $<0.0001$ \\
Paralytic Ileus & 25.23 & 21.53 & $<0.0001$ \\
Mortality, Overall (\%) & 2.2 & 0.7 & $<0.0001$ \\
LOS (days) & & & 0.1893 \\
Median & 8 & 8 & \\
IQR & $7-12$ & $7-11$ & $<0.0001$ \\
Cost & & & \\
Median & $\$ 25,184$ & $\$ 26,817$ & \\
IQR & $\$ 18,778-\$ 35,200$ & $\$ 20,442-\$ 36,999$ & \\
\hline
\end{tabular}

Table 4

Multivariate logistic regression adjusted for age, gender, comorbidities, and hospital characteristics

\begin{tabular}{lccc}
\hline Variable & $\begin{array}{c}\text { OR for IC } \\
\text { compared to CD }\end{array}$ & $95 \%$ CI & $p$-value \\
\hline Complications, Overall (\%) & 1.07 & $1.01-1.12$ & 0.0185 \\
Complications, Infectious (\%) & 1.13 & $1.05-1.22$ & 0.002 \\
Mortality, Overall (\%) & 1.87 & $1.38-2.52$ & $<0.0001$ \\
LOS (days) & 1.02 & $0.99-1.05$ & 0.1893 \\
Cost & 0.95 & $0.92-0.98$ & 0.0008 \\
\hline
\end{tabular}

cystectomy (RC), no consensus has been reached. Patients ideally receive that which provides them optimal cancer control, minimizes complications, and confers the highest quality of life following the procedure [8]. Although no definitive recommendations exist, many experts suggest continent diversions be used when feasible [9]. For example, the 2017 American Urological Association muscle-invasive bladder cancer guideline stresses the importance of discussing both IC and CD with the patient [10]. Nonetheless, our analysis found that in recent years the utilization of continent diversions (CD) is significantly lower than that of ileal conduits (IC). While the number of CDs did statistically rise when comparing the start of our analysis in 2001 to the end in 2012, ultimately the number of CDs peaked in 2008 and has declined every year thereafter. These results are consistent with and expand upon previous reports $[2,11,12]$. One possible explanation for the decline in CDs is the increasing use of robotics to perform RC, which has traditionally been performed with an open approach. In a cohort of 2,525 patients undergoing RC from October 2008-December 2012,
Pak et al. found that $75.8 \%$ were performed openly vs. $24.2 \%$ robotically. By 2012 , there was a $28.9 \%$ increase in the number of robotic procedures being performed [13]. While there is no analysis of urinary diversion type in this study, we surmise that most robotic RCs are performed with an IC rather than a $\mathrm{CD}$, as CDs are more challenging to create. Moreover, although surgeries for continent diversions may be more challenging, reimbursements are only approximately \$200 higher for these procedures. Therefore, urologists may be less inclined to offer or perform continent diversions [14]. Our results highlight the need for further understanding of the causes of such disparity given our current knowledge of the two surgical approaches.

CDs are often thought as a more technically complex procedure requiring longer surgical time, and many surgeons may not be adequately trained to perform them with consistent success. An examination of urology residency training programs found that the mean number of continent and incontinent urinary diversions performed by a resident throughout their training was 6.7 and 9.5, respectively [15]. Moreover, 
only $30 \%$ of all graduating urology residents performed more than 20 urinary diversions of any type. As a consequence, many surgeons may have a lack of comfort in not only performing continent diversions, but also in the post-operative management [16]. Likewise, Gore et al. evaluated 3611 patients from the SEER-Medicare database who underwent radical cystectomy between 1992-2000 and showed that having surgery at an NCI-designated cancer center resulted in a 5-fold increased likelihood of having a continent diversion (OR 5.50, $p<0.001)$ [17]. Surgeons at these centers are more likely to be fellowship trained and thus presumably performed more of these procedures during training. Teaching hospitals, especially NCI designated cancer centers, might also have nursing and support staff that is more familiar with CDs, allowing surgeons to feel more comfortable performing them. Our finding that urban teaching hospitals performed the most CDs when compared to rural and urban non-teaching hospitals may similarly reflect these ideas and the growing regionalization of radical cystectomy $[18,19]$.

In general, the morbidity associated with RC is well-established [20]. Although there may be a perception that CD increases postoperative complications, our findings did not support this hypothesis. Our analysis showed that there was a significantly higher rate of overall complications $(52.85 \%$ vs. $44.66 \% ; p<0.0001)$ and mortality $(2.17 \%$ vs. $0.67 \%$; $p<0.0001)$ in the IC than CD groups, respectively. However, a selection bias towards patients who are younger and healthier at baseline may account for these findings. We found that younger patients (age 40-59) underwent the highest proportion of CDs (40.3\%), suggesting that surgeons may perceive younger patients to be better candidates for these procedures or that younger patients may be more motivated to undergo CD. Nonetheless, IC was still performed at a significantly higher rate than expected even among those in this age group $(79.5 \%$ vs. $20.5 \%$; $p<0.0001$ ), suggesting a potential underutilization of CD. Interestingly, while many studies seem to agree that age is a risk factor for perioperative morbidity associated with RC [20], there is little evidence that older patients who receive a $\mathrm{CD}$ are at significantly higher risk of complications. Clark et al. showed there was no difference in complications or mortality when comparing patients with an age $>70$ who received either IC or CD [21]. Likewise, Sogni and colleagues retrospectively analyzed 85 patients with a median age of 78 who underwent RC, and found no significant difference in perioperative complications between those who received an IC or CD [22]. Additionally, previous studies have found that patients with a lower Charlson Comorbidity Index (CCI) [11] and American Society of Anesthesiologist class (ASA) [5] were more likely to undergo $\mathrm{CD}$. It is possible that lower complication rates observed in patients undergoing $\mathrm{CD}$ may be attributable to confounding variables including patient age and preoperative functional status. However, after controlling for age, gender, comorbidities, and hospital characteristics, we observed that patients receiving an IC were 1.06 times more likely to have any complication and 1.87 times more likely to experience in-hospital mortality compared to CD. Taken together, these results imply that with careful patient selection, similar rates of complications can be expected in patients undergoing IC or CD, regardless of age.

Patient gender and race may also influence the choice of urinary diversion. Surprisingly, males were greater than 2 times more likely to receive a $\mathrm{CD}$, while White and Hispanic patients were more likely than African Americans to receive a CD. An increased incidence of voiding dysfunction following orthotopic neobladder creation in females has been described which may cause some surgeons to reserve its use to select female patients [23, 24]. On the other hand, the racial disparity in urinary diversion utilization may be a result of assorted socioeconomic factors influencing patients' access to healthcare. Roghmann et al. found that privately insured patients and those residing in ZIP codes with a median income of $\geq \$ 45,000$ were likely to receive a $C D$ upon multivariate analysis [11]. Maurice et al. reported that patients with an income $>\$ 63,000$ were 2.9 and 2.2 times more likely to receive a continent cutaneous reservoir or orthotopic neobladder, respectively, over an ileal conduit. They suggest that patients with lower incomes may lack the social supports and resources to maintain continent diversions. Also, patients in low-income groups are less likely to be formally educated; these authors commented that patients may lack the ability to understand and perform straight catheterization, a necessary skill in those electing to have a continent diversion [14]. This is interesting given our finding that the cost of $\mathrm{CD}$ was significantly higher than IC ( $\$ 26,817$ vs. $\$ 25,184 ; p<0.0001)$. It is unclear whether these factors directly influence the choice of which UD to perform. Further investigation of the role of socioeconomic status on this population and physician reimbursement patterns are warranted, and any disparities should be addressed. 
The current study collected data from the NIS in order to investigate the trends in utilization of ileal conduits and continent diversions, including orthotopic neobladders, following radical cystectomy. The NIS comprises information from more than 7 million hospital stays per year, making it a robust tool to estimate trends in healthcare utilization. To the best of our knowledge, this analysis encompasses the largest and most up to date sample of the NIS database. However, it is not without its limitations. Clinical information such as functional status, cancer stage, and other tumor characteristics are not made available through the NIS database. Thus any role more aggressive disease phenotypes or other common clinical determinants have in influencing the choice of urinary diversion cannot be elucidated [12]. Results were not stratified by surgical approach (i.e. robotic versus open), as the current procedural terminology (CPT) code for robotic cystectomy is notably non-specific ("Unlisted laparoscopy procedure, bladder") and limits proper analysis. We are also unable to account for patient preference influencing any observable trends. Finally, long-term oncologic outcomes and complications after hospital discharge are also not available, both of which are important in any discussion. As the NIS only captures data associated with the index hospitalization time period, we did not assess the long-term overall complication rates of the different procedures. Nonetheless, it is important for providers to properly counsel patients on all aspects of the recovery of these procedures and perioperative complication rates have not been previously well documented.

\section{CONCLUSION}

Our current study presents a robust analysis of the NIS database showing a declining trend in the usage of CDs in recent years. Patients of all ages are more likely to receive IC than $\mathrm{CD}$. Gender, racial, and geographic disparities exist among those receiving CDs. CDs are associated with lower rates of in-hospital complications and mortality, though in-hospital costs are higher. Possible reasons for declining incidence of continent diversions might include physician reimbursement, length of surgical time, increasing use of robotic surgery and physician's counseling of patients regarding diversion options. Prospective studies are needed to further elucidate the many potential factors resulting in disparate use of urinary diversions.

\section{SOURCES OF FUNDING}

This research did not receive any specific grant from funding agencies in the public, commercial, or not-for-profit sectors. This work is supported in part by a grant from the National Cancer Institute (P30CA072720).

\section{CONFLICT OF INTEREST}

Sammy E. Elsamra is an Intuitive Surgical ${ }^{\circledR}$ robotic proctor. All other authors have no conflicts of interest to report.

\section{REFERENCES}

[1] Siegel RL, Miller KD, Jemal A. Cancer Statistics, 2017. CA Cancer J Clin 2017;67(1):7-30.

[2] Gore JL, Litwin MS, Urologic Diseases in America P. Quality of care in bladder cancer: Trends in urinary diversion following radical cystectomy. World J Urol 2009;27(1): 45-50.

[3] Goldberg H, Baniel J, Mano R, Rotlevy G, Kedar D, Yossepowitch O. Orthotopic neobladder vs. ileal conduit urinary diversion: A long-term quality-of-life comparison. Urol Oncol 2016;34(3):121 e1-7.

[4] Horovitz D, Turker P, Bostrom PJ, Mirtti T, Nurmi M, Kuk $\mathrm{C}$, et al. Does patient age affect survival after radical cystectomy? BJU Int 2012;110(11 Pt B):E486-93.

[5] Lowrance WT, Rumohr JA, Clark PE, Chang SS, Smith JA Jr., Cookson MS. Urinary diversion trends at a high volume, single American tertiary care center. J Urol 2009;182(5): 2369-74.

[6] Jahnson S, Damm O, Hellsten S, Holmang S, Liedberg F, Ljungberg B, et al. Urinary diversion after cystectomy for bladder cancer: A population-based study in Sweden. Scand J Urol Nephrol 2010;44(2):69-75.

[7] Collins JW, Tyritzis S, Nyberg T, Schumacher MC, Laurin $\mathrm{O}$, Adding $\mathrm{C}$, et al. Robot-assisted radical cystectomy (RARC) with intracorporeal neobladder - what is the effect of the learning curve on outcomes? BJU Int 2014;113(1): 100-7.

[8] Hautmann RE. Urinary diversion: Ileal conduit to neobladder. J Urol 2003;169(3):834-42.

[9] Hautmann RE, Abol-Enein H, Lee CT, Mansson W, Mills $\mathrm{RD}$, Penson DF, et al. Urinary diversion: How experts divert. Urology 2015;85(1):233-8.

[10] Chang SS, Bochner BH, Chou R, Dreicer R, Kamat AM, Lerner SP, et al. Treatment of Non-Metastatic MuscleInvasive Bladder Cancer: AUA/ASCO/ASTRO/SUO Guideline. J Urol. 2017.

[11] Roghmann F, Becker A, Trinh QD, Djahangirian O, Tian Z, Meskawi M, et al. Updated assessment of neobladder utilization and morbidity according to urinary diversion after radical cystectomy: A contemporary USpopulation-based cohort. Can Urol Assoc J 2013;7(9-10): E552-60.

[12] Kim SP, Shah ND, Weight CJ, Thompson RH, Wang JK, Karnes RJ, et al. Population-based trends in urinary diversion among patients undergoing radical cystectomy for bladder cancer. BJU Int 2013;112(4):478-84. 
[13] Pak JS, Lee JJ, Bilal K, Finkelstein M, Palese MA. Utilization Trends and Short-term Outcomes of Robotic Versus Open Radical Cystectomy for Bladder Cancer. Urology 2017;103:117-23.

[14] Maurice MJ, Kim SP, Abouassaly R. Socioeconomic status is associated with urinary diversion utilization after radical cystectomy for bladder cancer. Int Urol Nephrol 2017;49(1):77-82.

[15] Chang SS, Smith JA Jr., Herrell SD, Cookson MS. Assessing urinary diversion experience in urologic residency programs-are we adequately training the next generation? J Urol 2006;176(2):691-3.

[16] Skinner EC. Choosing the right urinary diversion: Patient's choice or surgeon's inclination? Urol Oncol 2011;29(5): 473-5.

[17] Gore JL, Saigal CS, Hanley JM, Schonlau M, Litwin MS, Urologic Diseases in America P. Variations in reconstruction after radical cystectomy. Cancer 2006;107(4):729-37.

[18] Smaldone MC, Simhan J, Kutikov A, Canter DJ, Starkey R, Zhu F, et al. Trends in regionalization of radical cystectomy in three large northeastern states from 1996 to 2009. Urol Oncol 2013;31(8):1663-9.

[19] Hollenbeck BK, Taub DA, Miller DC, Dunn RL, Montie JE, Wei JT. The regionalization of radical cystectomy to specific medical centers. J Urol 2005;174(4 Pt 1):1385-9; discussion 9.
[20] Novara G, Catto JW, Wilson T, Annerstedt M, Chan K, Murphy DG, et al. Systematic review and cumulative analysis of perioperative outcomes and complications after robotassisted radical cystectomy. Eur Urol 2015;67(3):376-401.

[21] Clark PE, Stein JP, Groshen SG, Cai J, Miranda G, Lieskovsky G, et al. Radical cystectomy in the elderly: Comparison of survival between younger and older patients. Cancer 2005;103(3):546-52.

[22] Sogni F, Brausi M, Frea B, Martinengo C, Faggiano F, Tizzani A, et al. Morbidity and quality of life in elderly patients receiving ileal conduit or orthotopic neobladder after radical cystectomy for invasive bladder cancer. Urology 2008; 71(5):919-23

[23] Anderson CB, Cookson MS, Chang SS, Clark PE, Smith JA Jr., Kaufman MR. Voiding function in women with orthotopic neobladder urinary diversion. J Urol 2012;188(1): 200-4.

[24] Finley DS, Lee U, McDonough D, Raz S, deKernion J. Urinary retention after orthotopic neobladder substitution in females. J Urol 2011;186(4):1364-9. 